\title{
Similar Improvements in Patient-Reported Outcomes Among Rheumatoid Arthritis Patients Treated with Two Different Doses of Methotrexate in Combination with Adalimumab: Results From the MUSICA Trial
}

\author{
Gurjit S. Kaeley • Daryl K. MacCarter · Janak R. Goyal • \\ Shufang Liu • Kun Chen · Jennifer Griffith · Hartmut Kupper • \\ Vishvas Garg • Jasmina Kalabic \\ Received: December 6, 2017 / Published online: March 24, 2018 \\ (C) The Author(s) 2018
}

\section{ABSTRACT}

Introduction: In patients with rheumatoid arthritis (RA), combination treatment with

Enhanced content To view enhanced content for this article go to https://doi.org/10.6084/m9.figshare. 5947891.

Electronic supplementary material The online version of this article (https://doi.org/10.1007/s40744018-0105-7) contains supplementary material, which is available to authorized users.

G. S. Kaeley ( $\square)$

University of Florida College of Medicine,

Jacksonville, FL, USA

e-mail: gurjit.kaeley@jax.ufl.edu

D. K. MacCarter

Coeur d'Alene Arthritis Clinic, Coeur d'Alene, ID, USA

J. R. Goyal

Raritan Bay Medical Center, Perth Amboy, NJ, USA

S. Liu · K. Chen · J. Griffith · V. Garg

AbbVie Inc., North Chicago, IL, USA

H. Kupper · J. Kalabic

AbbVie Deutschland GmbH and Co. KG.,

Ludwigshafen, Germany

Present Address:

D. K. MacCarter

North Valley Hospital, Whitefish, MT, USA

Present Address:

S. Liu

Astellas Pharma, Northbrook, IL, USA methotrexate (MTX) and adalimumab is more effective than MTX monotherapy. From the patients' perspective, the impact of reduced MTX doses upon initiating adalimumab is not known. The objective was to evaluate the effects of low and high MTX doses in combination with adalimumab initiation on patient-reported outcomes (PROs), in MTX-inadequate responders (MTX-IR) with moderate-to-severe RA.

Methods: MUSICA was a randomized, doubleblind, controlled trial evaluating the efficacy of 7.5 or $20 \mathrm{mg} /$ week MTX, in combination with adalimumab for 24 weeks in MTX-IR RA patients receiving prior $\mathrm{MTX} \geq 15 \mathrm{mg} /$ week for $\geq 12$ weeks. PROs were recorded at each visit, including physical function, health-related quality-of-life, work productivity, qualityof-sleep, satisfaction with treatment medication, sexual impairment due to RA, patient global assessment of disease activity (PGA), and patient pain. Last observation carried forward was used to account for missing values.

Results: At baseline, patients in both MTX dosage groups had similar demographics, disease characteristics, and PRO scores. Overall, initiation of adalimumab led to significant improvements from baseline in the PROs assessed for both MTX dosage groups. Improvements in presenteeism from baseline were strongly correlated with corresponding improvements in SF-36 (vitality), pain, and physical function. Physical and mental wellbeing had a good correlation with improvement 
in sleep. Overall, improvements in disease activity from baseline were correlated with improvements in several PROs.

Conclusions: The addition of adalimumab to MTX in MTX-IR patients with moderate-tosevere RA led to improvements in physical function, quality-of-life, work productivity, quality of sleep, satisfaction with treatment medication, and sexual impairment due to RA, regardless of the concomitant MTX dosage.

Funding: AbbVie.

Trial Registration: Clinicaltrials.gov identifier, NCT01185288.

Keywords: Adalimumab; treatment; Dose reduction; Methotrexate; Patient-reported outcomes; Rheumatoid arthritis

\section{INTRODUCTION}

Rheumatoid arthritis (RA) is a chronic, progressive, autoimmune disease characterized by inflammation of the synovial membrane of the joints. Uncontrolled inflammation can lead to erosion of the bone and cartilage, permanent damage and functional impairment of the joints, and may have systemic effects on the cardiovascular system, lungs, and skin. RA affects approximately $0.5-1.0 \%$ of the general population, with women being affected more frequently than men [1]. RA is globally ranked among the top 50 disabilities and the disabilityadjusted life years increased from 3.3 million in 1990 to 4.8 million in 2010 [2], which indicates an increased loss of years due to poor health, disability, or death.

Patients with RA experience a reduced quality of life compared with the general population, and the disease affects patients physically as well as psychologically. Functional disability may result in a reduced ability to work and participate in daily activities. RA patients also experience an increased prevalence of depression [3], and reduced sleep quality compared to the general US population [4]. Reduced ability to do one's job, fewer hours worked, lower productivity, and loss of paid employment place an economic burden on both patients and society [5-8].

RA can be effectively controlled using synthetic or biological disease-modifying antirheumatic drugs (DMARDs), alone or in combination. The long-term goal of RA treatment is to maximize patients' quality-of-life [9], and the value of the patient's perspective in assessing treatment efficacy is being increasingly recognized by collecting data on patient-reported outcomes (PROs). PROs can measure intangible effects on quality of life, pain, and fatigue, thus complementing traditional physician assessments. Recognizing the relevance of PROs to the patient, there has been a trend towards regulators requesting more of this information.

Methotrexate (MTX) is a recommended firstline treatment for patients with moderate-toseverely active RA $[9,10]$. In the case of patients who have an inadequate response to MTX (MTXIR) [11], treatment may be modified by addition of another synthetic DMARD or a TNF-inhibitor, such as adalimumab. Although adalimumab and MTX are known to have synergistic effects, the MUSICA trial aimed to evaluate the ideal dose of MTX needed in this setting. In particular, MUSICA evaluated the effects of initiating adalimumab treatment in combination with reduced (7.5 mg/week) and high (20 mg/week) MTX doses in MTX-IR RA patients, who were receiving prior MTX (at least $15 \mathrm{mg} /$ week) [12]. The primary endpoint was week-24 mean 28 -joint disease activity score based on C-reactive protein (DAS28[CRP]) to test non-inferiority of lowdosage MTX to high-dosage MTX. Here we report the effects of treatment on PROs such as physical functioning, quality of life, work productivity and activity impairment, sleep quality, sexual impairment due to RA, and satisfaction with medication.

\section{METHODS}

\section{Patients and Study Design}

MUSICA (NCT00185288) was a randomized, double-blind, controlled, 24-week trial enrolling 309 patients, at least 18 years of age, with active RA symptoms. At baseline, patients were 
required to have DAS28(CRP) $\geq 3.2$. At screening or baseline, patients must have had $\geq 5$ swollen joints out of 66 joints assessed and $\geq 5$ tender joints out of 68 joints assessed. Patients must have received a stable MTX dose of at least $15 \mathrm{mg}$ per week for at least 12 weeks prior to screening, and must have had an inadequate response to MTX. Enrolled patients were required to be adalimumab-naive and up to $10 \%$ of patients were allowed to have prior treatment with one biologic. Patients were stratified based on incoming MTX dosage and randomized (1:1) to receive blinded MTX, at either 7.5 or $20 \mathrm{mg} /$ week, in combination with open-label $40 \mathrm{mg}$ of adalimumab every other week. Clinical efficacy measurements were performed at baseline and weeks 4, 8, 12, 16, 20, and 24 . All patients provided informed consent and the study was conducted in accordance with the protocol, International Conference on Harmonization and with the Helsinki Declaration of 1964 as revised in 2013.

\section{Patient-Reported Outcomes}

The PROs were recorded at each study visit, from baseline to week 24, using an Electronic PRO (ePRO) tool (CRF Health, Lansdale, PA, USA), or paper questionnaires. Physical functioning was assessed using the health assessment questionnaire-disability index (HAQ-DI), which measures the degree of difficulty experienced by patients while performing common activities such as dressing, grooming, hygiene, arising, eating, walking, reaching, gripping, and doing chores. HAQ-DI scores range from 0 (no disability) to 3 (severe disability); the minimum clinically important difference (MCID) is defined as a decrease from baseline of $\geq 0.22$ [13].

Health-related quality of life (HRQoL) was measured using the Short-Form 36 version 2 (SF$36 \mathrm{v} 2)$ questionnaire, which is composed of eight subdomains: physical functioning, role limitations due to physical health, bodily pain, general health perceptions, vitality, social functioning, role limitations due to emotional health, and mental health. Further, these eight subdomains of SF-36 can be translated into two summary scores, i.e., physical component summary (PCS) and mental component summary (MCS), which are normalized to US general population level with a mean of $50 \pm 10$. Together, the normalized scores of PCS and MCS range from 0 to 100; higher scores indicate better health. The MCID are defined as an increase of 5 points for the summary SF-36 score or 2.5 points for the PCS and MCS individually $[14,15]$.

Work productivity and regular activity impairment were measured using the work productivity and impairment (WPAI) questionnaire [16], which is composed of six questions relating to presenteeism (percent impairment while working), absenteeism (percent work time missed or working hours missed due to RA), overall impairment (absenteeism plus presenteeism), regular activities impairment (percentage activity impairment). The measured impairment ranges from 0 to $100 \%$, with higher scores indicating greater impairment. In this study, work absenteeism-percent time is expressed in terms of working hours missed due to RA [17].

Satisfaction with treatment medication was assessed using the Treatment Satisfaction Questionnaire for Medication (TSQM) Version 1.4 , which is composed of 14 items to derive four subdomains: efficacy, convenience, general satisfaction, and side effects. Scores range from 0 to 100 , with a higher score indicating greater satisfaction. Patient Global Assessment of disease activity (PGA) and Patient Global Assessment of pain (PGA-pain) were assessed using a visual analog scale (VAS) from 0 to $100 \mathrm{~mm}$.

\section{Statistical Analysis}

Two types of comparisons were conducted. First, the mean change for each PRO from baseline to week 24 was calculated and tested using $t$ test separately for the low- and highdosage MTX groups. Second, differences in mean changes in PROs at week 24 between the low- and high-dosage MTX groups were compared using analysis of covariance (ANCOVA) including baseline values in the model. Last observation carried forward (LOCF) was used to 
account for missing values. The relationship between improvements from baseline to week 24 in fatigue (represented by SF-36-vitality), PGA-pain or HAQ-DI and corresponding improvement in WPAI-presenteeism were assessed by Pearson's correlation coefficients. The correlation of physical or mental well-being with sleep was assessed by calculating Pearson's coefficient for SF-36 PCS or SF-36 MCS with each individual subdomain of the MOS Index II. The correlation of disease activity with HAQ-DI, SF-36 PCS/MCS, TSQM, and WPAI was also assessed by Pearson's coefficients.

\section{RESULTS}

Three-hundred and nine patients were randomized in MUSICA, 154 patients in the $7.5 \mathrm{mg} /$ week MTX plus adalimumab group, and 155 patients in the $20 \mathrm{mg} /$ week MTX plus adalimumab group. The mean age at baseline was 54.8 years, and patients were predominantly female $(74.8 \%)$. Patients had established RA (mean disease duration of 5.3 years) and high disease activity, indicated by a mean DAS28(CRP) score of 5.8. The mean duration of MTX treatment prior to the study was 1.5 years.
There were no significant differences in baseline demographics, disease characteristics, and PROs between the two MTX dosage groups (Table S1 in the supplementary material).

\section{Physical Function and Quality of Life}

Upon initiating treatment with adalimumab plus 7.5 or $20 \mathrm{mg}$ MTX/week, improvements were observed in both MTX dosage groups; most of the improvement occurred rapidly in the first 12 weeks and was maintained out to week 24 (Fig. 1a). At week 24, both MTX dosage groups showed statistically significant and clinically meaningful improvements in HAQ-DI from baseline ( $p<0.001$, Fig. 1b) and met the MCID (decrease from baseline $\geq 0.22$ ); the improvements from baseline in HAQ-DI were statistically not significantly different for the two MTX dosage groups.

Over 24 weeks of treatment, improvements from baseline were observed in the SF-36 PCS and MCS in both MTX dosage groups (Fig. 2a, c). At week 24 , patients in both dosage groups showed a significant $(p<0.001)$ change from baseline, which was clinically meaningful (reaching the MCID, an increase $>2.5$ ) for PCS
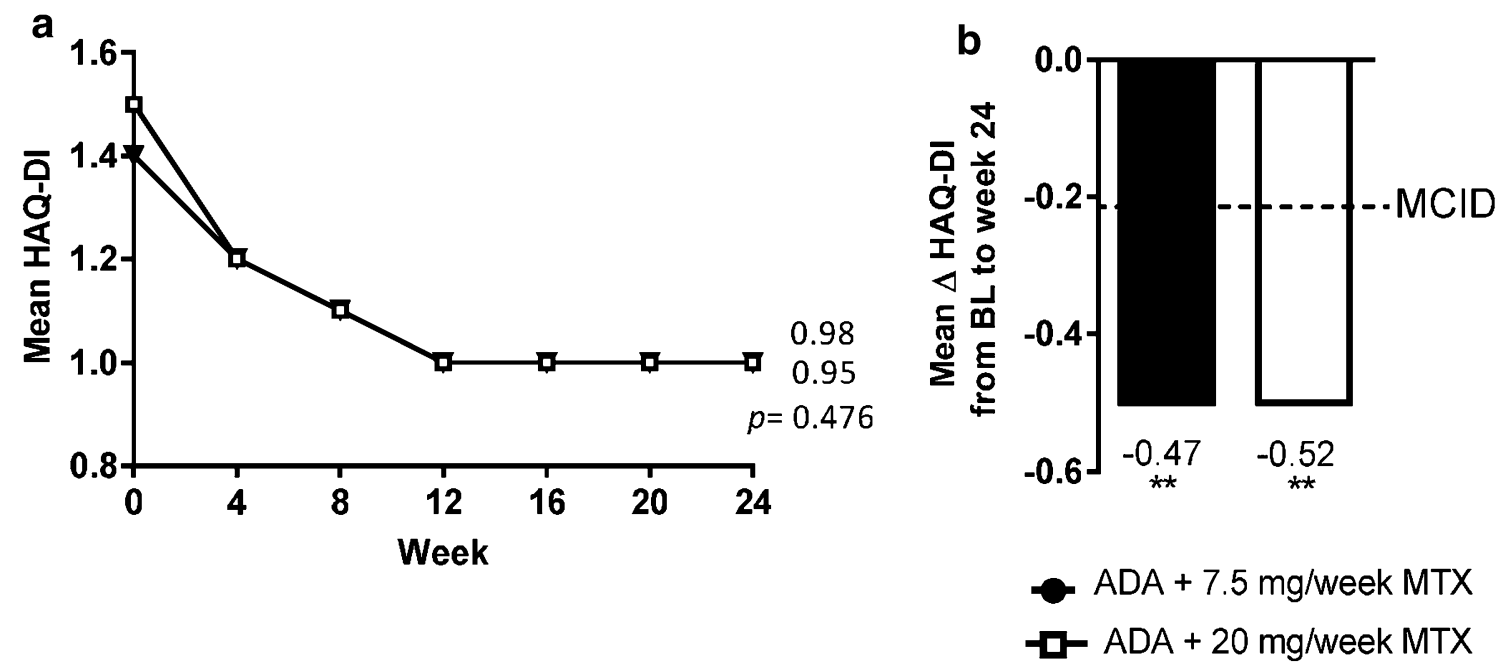

Fig. 1 a Physical function (HAQ-DI) over time. $p$ values for comparison between MTX dosage groups at week 24 (ANCOVA). b Mean change in physical function from baseline to week $24 .{ }^{* *} p<0.001$, comparison between scores at baseline and at week $24(t$ test $)$ for each MTX

dosage group. Reprinted from the 2014 ACR/ARHP Annual Meeting held November 14-19, 2014. The American College of Rheumatology does not guarantee, warrant, or endorse any commercial products or services 


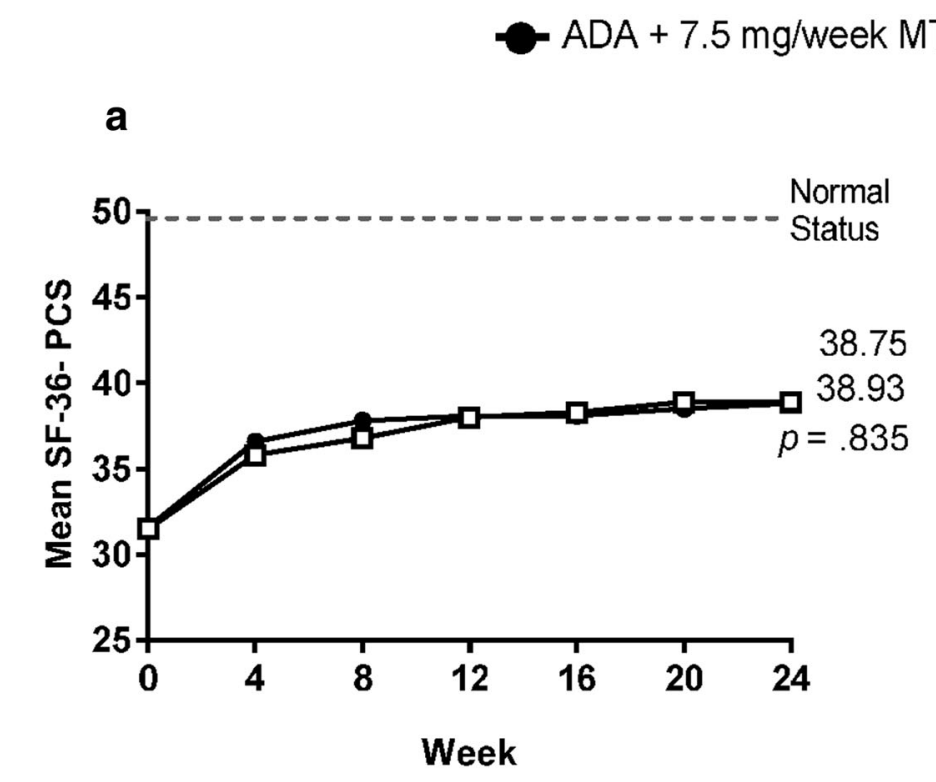

\section{$\square$ ADA $+20 \mathrm{mg} /$ week MTX}

b
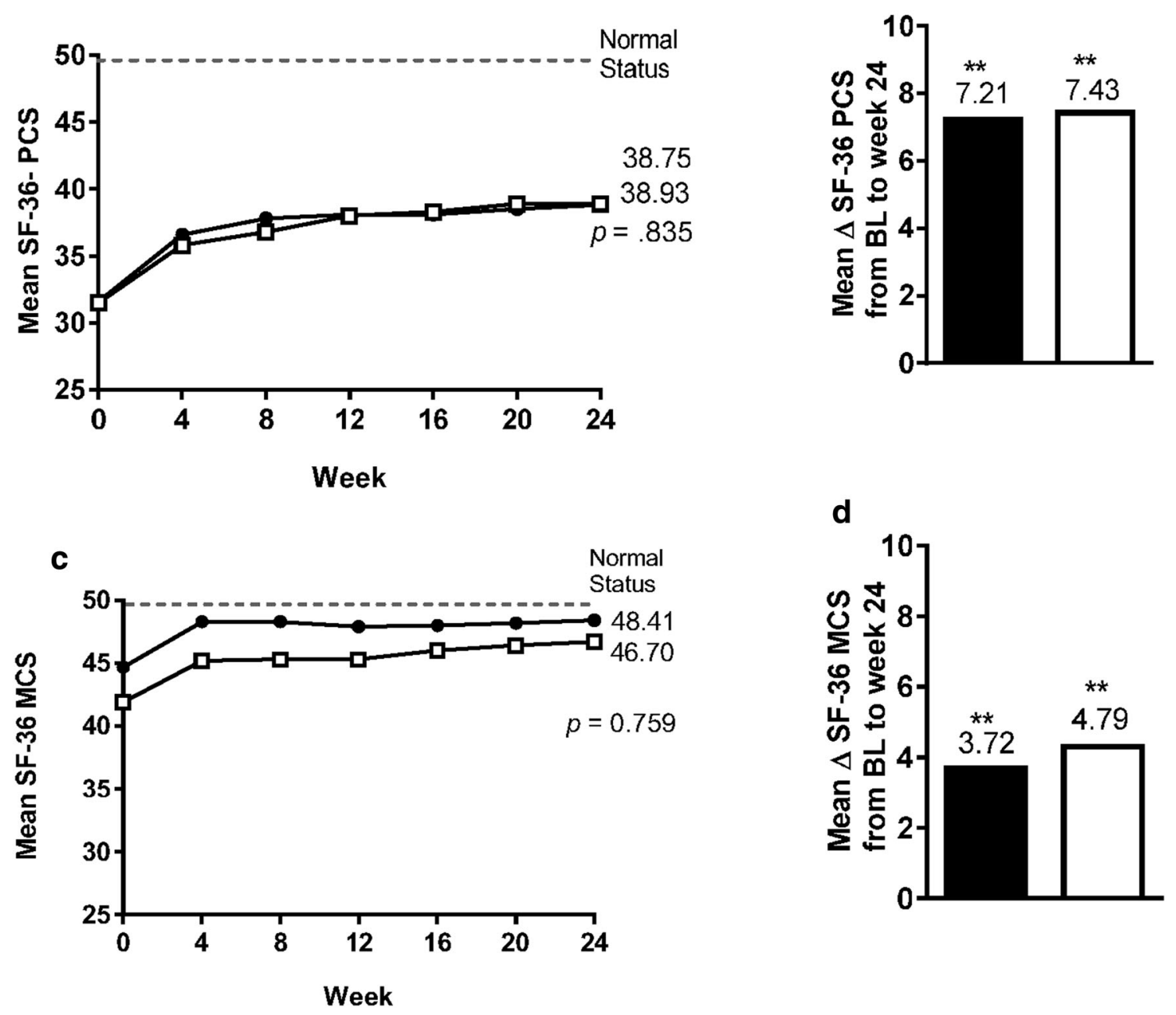

Fig. 2 a SF-36 PCS over time and b mean change in SF-36 PCS from baseline to week 24. c SF-36 MCS over time and d mean change in SF-36 MCS from baseline to week 24. $p$ values of comparison between MTX dosage groups at week 24 (ANCOVA) in a and c. $^{* *} p<0.001$, comparison

and MCS. Mean improvements from baseline to week 24 for SF-36 PCS and MCS (Fig. 2b, d) were numerically slightly higher for the $20 \mathrm{mg}$ MTX group. The MCS scores approached the normal population score $($ mean $=50)$. A moderate correlation between improvement in disease activity (measured by DAS28(CRP), and improvement in HAQ-DI $(r=0.56)$, SF-36 PCS $(r=-0.46)$ and MCS $(r=-0.33)$ at week 24, indicated that an improvement in disease between scores at baseline and at week 24 ( $t$ test) for each MTX dosage group in $\mathbf{b}$ and $\mathbf{d}$. Reprinted from the 2014 ACR/ARHP Annual Meeting held November 14-19, 2014. The American College of Rheumatology does not guarantee, warrant, or endorse any commercial products or services

activity was moderately associated with an improvement in physical function as well as physical and mental well-being.

\section{Work Productivity and Activity Impairment}

Upon initiating treatment with adalimumab plus MTX, in general, in the subdomains of WPAI, rapid improvement was observed by 
ADA + 7.5 mg/week MTX

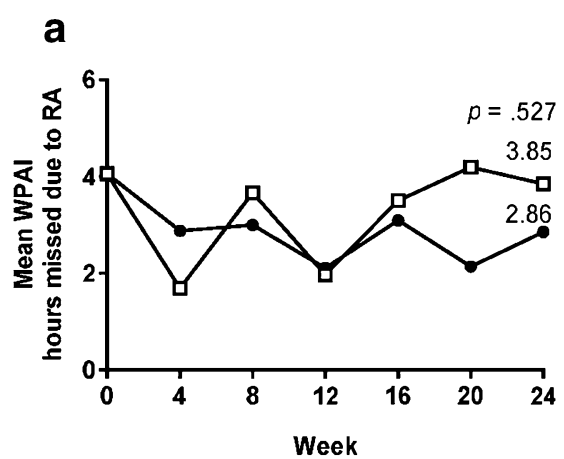

C

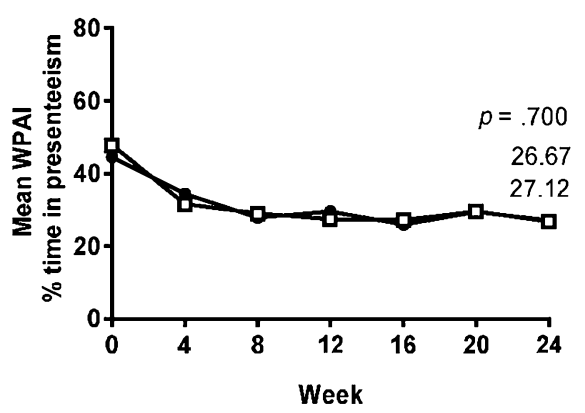

e

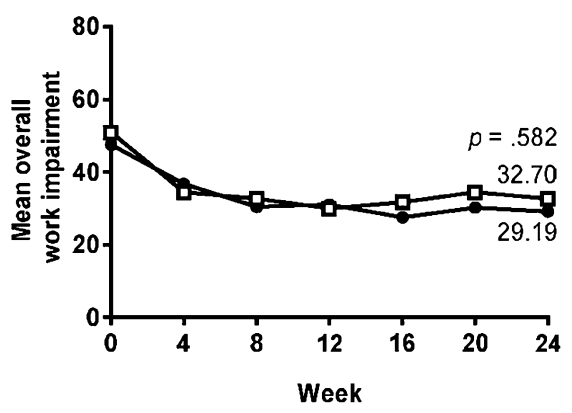

g

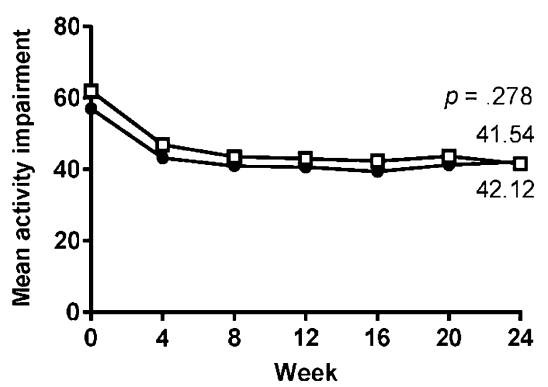

ADA + $20 \mathrm{mg} /$ week MTX

b

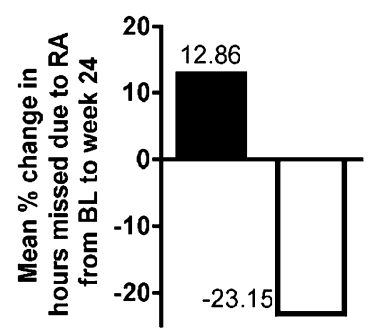

d

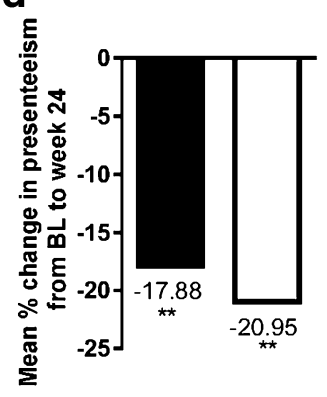

f

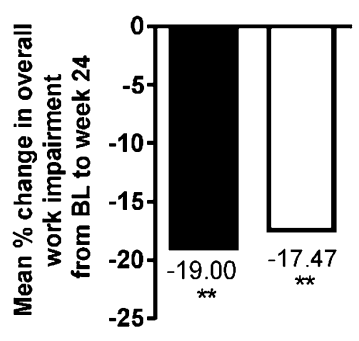

h

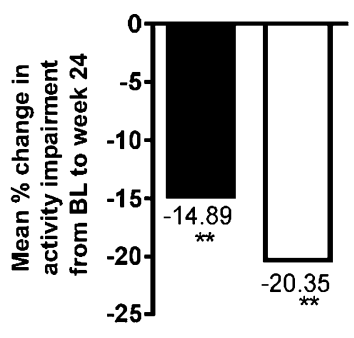


4Fig. 3 a WPAI hours missed due to RA overtime and b mean change in hours missed due to RA from baseline to week 24. c WPAI- \% time in presenteeism over time and d mean change in $\%$ time in presenteeism from baseline to week 24. e Overall work impairment over time and $\mathbf{f}$ mean change in overall work impairment from baseline to week 24. g Overall activity impairment over time and $\mathbf{h}$ mean change in overall activity impairment from baseline to week 24. $p$ values of comparison between dosage groups at week 24 (ANCOVA) in a, c, e and g. ${ }^{* *} p<0.001$, comparison between scores at baseline and at week 24 ( $t$ test) for each MTX dosage group in $\mathbf{b}, \mathbf{d}, \mathbf{f}$ and h. Reprinted from the 2014 ACR/ARHP Annual Meeting held November 14-19, 2014. The American College of Rheumatology does not guarantee, warrant, or endorse any commercial products or services

week 4 and maintained to week 24 (Fig. 3a, c, e, g). After 24 weeks of treatment, statistically significant improvements from baseline were observed in presenteeism, overall work impairment, and activity impairment in both MTX dosage groups $(p<0.001$, Fig. $3 d, f, h)$. In the case of working hours missed due to RA, the improvement from baseline to week 24 was not statistically significant (Fig. 3b). The mean improvement from baseline to week 24, for presenteeism, overall work impairment and activity impairment of the low- and high- MTX dosage groups were not statistically different from each other, although numerically slightly higher for the $20 \mathrm{mg}$ MTX group in all but overall work impairment. For both dosage groups, the improvements in WPAI- presenteeism from baseline to week 24 were moderately correlated with the corresponding improvements in fatigue [i.e., SF-36 (vitality) $r=0.51$ and 0.50 for low- and high-dosage MTX groups, respectively], and improvements in PGA-pain ( $r=0.59$ and 0.66, respectively). Improvements in presenteeism showed moderate-high correlation with improvements in HAQ-DI ( $r=0.59$ and 0.77) (Table S2 in the supplementary material). The improvement in disease activity after 24 weeks of treatment was also moderately correlated to the improvement in WPAI- overall impairment $(r=0.47)$ and WPAI- regular activities impairment $(r=0.40)$.

\section{Satisfaction with Medication}

After 24 weeks of treatment, improvements from baseline were observed in all TSQM subdomains, except for TSQM-convenience, with rapid improvement in the first 4 weeks, which was maintained out to week 24 (Fig. 4a, c, e, g). The improvements from baseline in TSQM-effectiveness and TSQM-global satisfaction subdomains were statistically significant for both MTX dosage groups ( $p<0.001$, Fig. $4 \mathrm{~b}, \mathrm{~d}, \mathrm{f}, \mathrm{h}$ ). The improvements from baseline to week 24 between the low- and high-MTX dosage groups were not statistically different for effectiveness, side effects, and global satisfaction. For each subdomain except TSQM convenience (which was lower), the changes from baseline to week 24 were numerically higher for the 20-mg MTX group. An improvement (reduction) in disease activity after 24 weeks of treatment was moderately correlated with an increase in general satisfaction $(-0.40)$ and efficacy $(-0.46)$ and weakly correlated with increased convenience $(-0.23)$ and reduced dissatisfaction with side effects $(-0.14)$.

\section{Sleep Quality and Correlation with Physical and Mental Well-being}

After 24 weeks of treatment, significant improvements $(p<0.001)$ from baseline were observed in most subdomains of the MOS-II, with the exception of the domain "awakened short of breath/headache". Most of the improvement was observed in the first 4 weeks and maintained to week 24 (Supplemental Figure 1). The improvement from baseline in MOS-II between the lowand high- MTX dosage groups was not statistically different.

A moderate negative correlation was observed between physical well-being measured by SF36-PCS, and some individual domains of the MOS Index: Sleep Index 9 ("trouble staying awake", $r=-0.55$ ), Sleep Index 6 ("feel drowsy", $\quad r=-0.52)$, sleep disturbance $(r=-0.51)$, daytime somnolence $(r=-0.46)$, awakened short of breath/headache $(r=-0.33)$. Perceived sleep adequacy showed a moderate positive correlation with physical 
ADA + 7.5 mg/week MTX

a

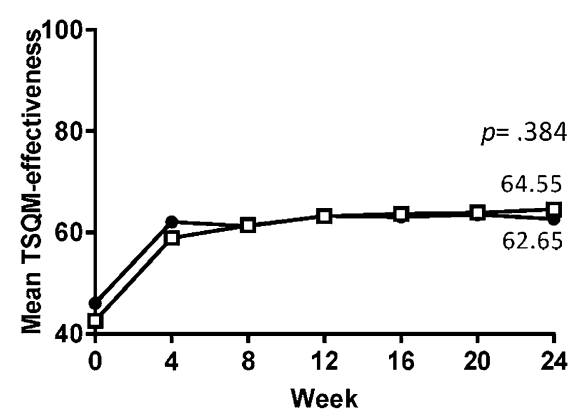

C

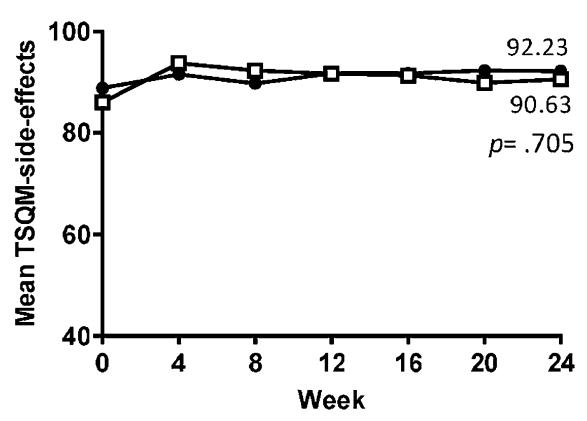

e

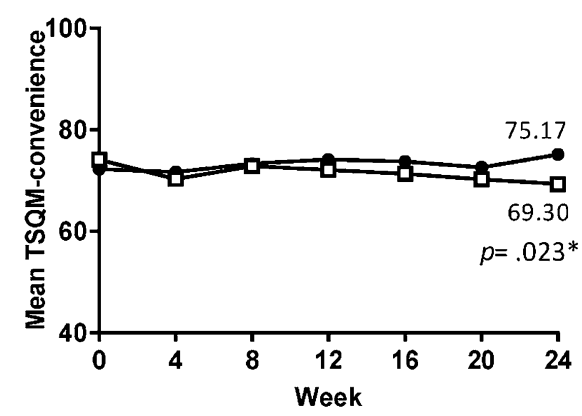

g

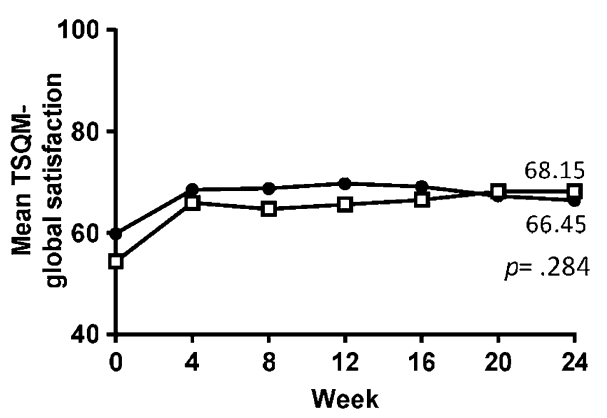

-ㄱ ADA + 20 mg/week MTX

b

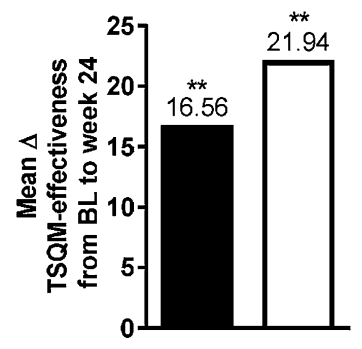

d

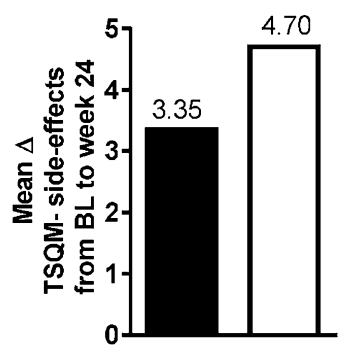

f

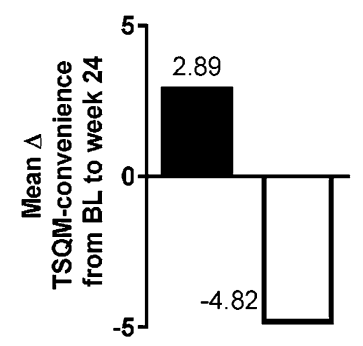

h

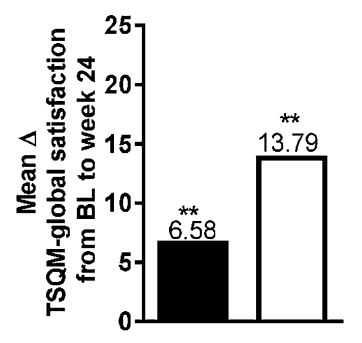


4Fig. 4 a Effectiveness over time. b Mean change in effectiveness from baseline to week 24. c Side effects over time. $\mathbf{d}$ Mean change in side effects from baseline to week 24. e Convenience over time. f Mean change in convenience from baseline to week 24. g Global satisfaction over time. $\mathbf{h}$ Mean change in global satisfaction from baseline to week 24. $p$ values of comparison between MTX dosage groups at week 24 (ANCOVA) in a, c, e and g. ${ }^{* *} p<0.001$, comparison between scores at baseline and at week 24 ( $t$ test) for each MTX dosage group in $\mathbf{b}$, d, $\mathbf{f}$ and $\mathbf{h}$

well-being $(r=0.36)$. Snoring and sleep quantity showed poor to no correlation with physical well-being ( $r=-0.19$ and 0.05 , respectively). Similar patterns were observed for correlations between mental well-being (measured by SF36MCS) and the individual domains of the MOS Index.

\section{Sexual Impairment Due to RA}

After 24 weeks of treatment, a statistically significant improvement in sexual impairment from baseline was observed for both MTX dosage groups, with the greatest improvement observed after 4-8 weeks of adalimumab initiation in a rapid manner (Supplemental Figure 2). At week 24, the mean improvements from baseline for the two MTX dosage groups were similar and not statistically different.

\section{DISCUSSION}

In the MUSICA trial, we found that patients with established RA who were MTX-IR reported significant improvements in several aspects of their overall quality of life when adalimumab treatment was initiated in combination with MTX. The data from MUSICA show that PROs in MTX-IR patients initiating adalimumab were not statistically different between the 7.5- or 20 mg MTX doses, indicating the feasibility from the patients' perspective of using a lower dose of MTX upon adalimumab initiation. In general, the PROs showed a rapid improvement 4 weeks after adalimumab initiation in combination with MTX, which was maintained out to 24 weeks, suggesting that the dominant effect on PROs within 6 months is likely due to adalimumab initiation.

In MUSICA, the primary endpoint of noninferiority of low-dosage MTX for DAS28(CRP) at week 24 compared to high dosage MTX was not met [12]. However, clinically significant improvements in disease activity were observed for both MTX dosage groups. Upon the addition of adalimumab, patients in both MTX dosage groups reported an improvement in physical functioning and achieved the MCID. Significant improvements were also observed in the physical and mental aspects of the health-related quality of life, as well as presenteeism, overall work impairment, and activity impairment.

The improvement in disease activity observed after 24 weeks was correlated with the improvements observed in physical function, physical and mental well-being, satisfaction with medication and impairment, which interfere with overall work productivity and regular activities.

The addition of adalimumab also led to lasting improvements in many aspects of sleep quality measured, including sleep disturbance, snoring, the quantity of sleep, somnolence during waking hours, and perceived sleep adequacy. The results are consistent with other studies demonstrating improved quality of sleep in patients with RA upon treatment with biologics $[4,18]$. Generally, less sleep disturbance, decreased feelings of drowsiness, and less trouble staying awake were moderately correlated with increased physical and mental well-being. In MUSICA, improvements in vitality, pain, and physical function were associated with improvements in work presenteeism.

A previous study on MTX intolerance in RA and PsA patients showed that $6.2 \%$ of these patients were intolerant to oral MTX [11]. The MUSICA data demonstrate that PROs in patients with established RA and MTX-IR were not affected, or only slightly affected, by the two different doses of MTX in combination with adalimumab. Rather than ceasing combination therapy and forgoing its benefits, these data indicate that there may be room for individualization of therapy in the event of MTX intolerance or toxicity. Obtaining PRO data may be useful for helping clinicians with making decisions on MTX dose adjustments during treatment with adalimumab. 
One limitation of the study was that patients entered the study with different previous dosages of MTX, ranging from 15 to $22 \mathrm{mg} /$ week. This may confound the results as patients may have already experienced some improvement due to prior treatment. The duration of treatment and data collection only extended out to 24 weeks. In MUSICA, we cannot rule out that persistent levels of polyglutamated MTX may have contributed to the lack of difference observed between the two MTX dosage groups for many of the PROs assessed. The findings in MUSICA are similar to those in the CONCERTO trial [19], where the initiation of treatment with a combination of adalimumab plus $2.5 / 5 / 10$ or $20 \mathrm{mg} /$ week MTX in treatment-naïve patients with early RA, led to similar improvements in a range of PROs including physical function, disease activity, sleep, and quality of life after 8 weeks [20]. The interpretation of the CONCERTO results was not affected by prior MTX usage as it was in MUSICA, which was conducted in MTX-IR patients. However, while not the primary objective of the MUSICA study design, the collection of PRO data in this clinically relevant MTX-IR population is of value, and the observations from this trial are relevant to a real-world clinical setting, where bDMARDs are added in combination in patients with inadequate response to MTX.

\section{CONCLUSIONS}

In the MUSICA trial, upon initiation of treatment with adalimumab, both low- and highdose MTX groups reported rapid and significant improvements in PROs from baseline to week 24 , which were associated with an improvement of disease activity. For many of the PROs, these improvements were comparable between the low- and high-dose MTX groups.

\section{ACKNOWLEDGEMENTS}

Funding. The work was sponsored by AbbVie Inc., North Chicago, USA. AbbVie sponsored the study (NCT01185288), contributed to its design, participated in the collection, analysis, and interpretation of the data, and in the writing, reviewing, and approval of the final version. Funding of article publication charges was provided by AbbVie. All authors had full access to all of the data in this study and take compete responsibility for the integrity of the data and the accuracy of the data analysis.

Authorship. All named authors meet the International Committee of Medical Journal Editors (ICMJE) criteria for authorship for this manuscript, take responsibility for the integrity of the work as a whole, and have given final approval to the version to be published. No deserving authors have been omitted.

Medical Writing and/or Editorial Assistance. Statistical support was provided by Peigang Li, Ph.D., of AbbVie; Medical writing support was provided by Naina Barretto, Ph.D., of AbbVie. AbbVie and the authors thank the patients who participated in the clinical trial and all study investigators for their contributions.

Disclosures. Gurjit S. Kaeley is a consultant for AbbVie. Daryl K. MacCarter has served on speakers bureaus and is a consultant for AbbVie. Janak R. Goyal is a consultant for AbbVie. Shufang Liu is a former AbbVie employee and may hold stock or stock options. Vishvas Garg is an AbbVie employee and may hold stock or stock options. Jenny Griffith is an AbbVie employee and may hold stock or stock options. Kun Chen is an AbbVie employee and may hold stock or stock options. Hartmut Kupper is an AbbVie employee and may hold stock or stock options. Jasmina Kalabic is an AbbVie employee and may hold stock or stock options.

Compliance and Ethics Guidelines. All patients provided informed consent and the study was conducted in accordance with the protocol, International Conference on Harmonization and with the Helsinki Declaration of 1964 as revised in 2013.

Data Availability. Qualified researchers may request access to the study data sets from 
AbbVie via the process defined on AbbVie.com under Clinical Trial Data and Information Sharing.

Open Access. This article is distributed under the terms of the Creative Commons Attribution-NonCommercial 4.0 International License (http://creativecommons.org/licenses/ by-nc/4.0/), which permits any noncommercial use, distribution, and reproduction in any medium, provided you give appropriate credit to the original author(s) and the source, provide a link to the Creative Commons license, and indicate if changes were made.

\section{REFERENCES}

1. Silman AJ. Work characteristics, demographic factors and clinical variables could predict work disability in rheumatoid arthritis. Clin Exp Rheumatol. 2001;19(3):247-8.

2. Cross M, Smith E, Hoy D, et al. The global burden of rheumatoid arthritis: estimates from the global burden of disease 2010 study. Ann Rheum Dis. 2014;73(7):1316-22.

3. Margaretten M, Julian L, Katz P, Yelin E. Depression in patients with rheumatoid arthritis: description, causes and mechanisms. Int J Clin Rheumtol. 2011;6(6):617-23.

4. Wolfe F, Michaud K, Li T. Sleep disturbance in patients with rheumatoid arthritis: evaluation by medical outcomes study and visual analog sleep scales. J Rheumatol. 2006;33(10):

$1942-51$

5. Puolakka K, Kautiainen H, Pekurinen M, et al. Monetary value of lost productivity over a five-year follow-up in early rheumatoid arthritis estimated on the basis of official register data on patients' sickness absence and gross income: experience from the FIN-RACo trial. Ann Rheum Dis. 2006;65(7):899-904.

6. Escorpizo R, Bombardier C, Boonen A, et al. Worker productivity outcome measures in arthritis. J Rheumatol. 2007;34(6):1372-80.

7. Barrett EM, Scott DG, Wiles NJ, Symmons DP. The impact of rheumatoid arthritis on employment status in the early years of disease: a UK community-based study. Rheumatology (Oxford). 2000;39(12):1403-9.

8. Birnbaum H, Shi L, Pike C, Kaufman R, Sun P, Cifaldi M. Workplace impacts of anti-TNF therapies in rheumatoid arthritis: review of the literature. Expert Opin Pharmacother. 2009;10(2):255-69.

9. Smolen JS, Landewe R, Breedveld FC, et al. EULAR recommendations for the management of rheumatoid arthritis with synthetic and biological disease-modifying antirheumatic drugs: 2013 update. Ann Rheum Dis. 2014;73(3):492-509.

10. Singh JA, Furst DE, Bharat A, et al. 2012 Update of the 2008 American College of Rheumatology recommendations for the use of disease-modifying antirheumatic drugs and biologic agents in the treatment of rheumatoid arthritis. Arthritis Care Res (Hoboken). 2012;64(5):625-39.

11. Calasan MB, van den Bosch OF, Creemers MC, et al. Prevalence of methotrexate intolerance in rheumatoid arthritis and psoriatic arthritis. Arthritis Res Ther. 2013;15(6):R217.

12. Kaeley GS, Evangelisto AM, Nishio MJ, Goss SL, Liu $\mathrm{S}$, Kalabic J, et al. Methotrexate dosage reduction adalimumab initiation: clinical and ultrasonographic outcomes from the randomized noninferiority MUSICA trial. J Rheumatol. 2016;43:1480-9.

13. Wells GA, Tugwell P, Kraag GR, et al. Minimum important difference between patients with rheumatoid arthritis: the patient's perspective. J Rheumatol. 1993;20:557-60.

14. Strand V, Boers M, Idzerda L, et al. It's good to feel better but it's better to feel good and even better to feel good as soon as possible for as long as possible. Response criteria and the importance of change at OMERACT 10. J Rheumatol. 2011;38(8):1720-7.

15. Strand V, Kingsbury SR, Woodworth $\mathrm{T}$, et al. OMERACT 10 Sharp Symposium: important findings in examination of imaging methods for measurement of joint damage in rheumatoid arthritis. J Rheumatol. 2011;38(9):2009-13.

16. Reilly MC, Zbrozek AS, Dukes EM. The validity and reproducibility of a work productivity and activity impairment instrument. Pharmacoeconomics. 1993;4(5):353-65.

17. Reilly A. WPAI Scoring. 2018. http://www. reillyassociates.net/WPAI_Scoring.html [cited http:// www.reillyassociates.net/WPAI_Scoring.html].

18. Zamarron C, Maceiras F, Mera A, Gomez-Reino JJ. Effect of the first infliximab infusion on sleep and alertness in patients with active rheumatoid arthritis. Ann Rheum Dis. 2004;63(1):88-90. 
19. Burmester GR, Kivitz AJ, Kupper H, et al. Efficacy and safety of ascending methotrexate dose in combination with adalimumab:the randomised CONCERTO trial. Ann Rheum Dis. 2015;74(6):1037-44.

20. Fleischmann RM, Kivitz A, van Vollenhoven RF et al. No differences in patient-reported outcomes by methotrexate dose among early rheumatoid Arthritis Patients Treates Concomitantly with Adalimumab: results from the CONCERTO trial (abstract). Ann Rheum Dis. 2013;72(suppl s3). 